\title{
VARIATIONAL METHODS FOR NONLINEAR ELLIPTIC EIGENVALUE PROBLEMS
}

\author{
BY FELIX E. BROWDER
}

Communicated September 24, 1964

In the present note, we give a simple general proof for the existence of solutions of the following two types of variational problems:

Problem A. To minimize $\int_{\Omega} F\left(x, u, \cdots, D^{m} u\right) d x$ over a subspace $V$ of $W^{m, p}(\Omega)$.

Problem B. To minimize $\int_{\Omega} F\left(x, u, \cdots, D^{m} u\right) d x$ for $u$ in $V$ with $\int_{\Omega} G\left(x, u, \cdots, D^{m-1} u\right) d x=c$.

The solution of the first problem yields a weak solution of a corresponding elliptic boundary-value problem for the Euler-Lagrange equation

$$
A u=\sum_{|\alpha| \leq m}(-1)^{|\alpha|} D^{\alpha} F_{p^{\alpha}}\left(x, u, \cdots, D^{m} u\right)=0 .
$$

From the solution of the second problem, we obtain a solution under corresponding boundary conditions of the nonlinear eigenvalue problem.

(2) $\quad A u=\lambda\left\{\sum_{|\beta| \leq m-1}(-1)^{|\beta|} G_{p \beta}\left(x, u, \cdots, D^{m-1} u\right)\right\}=\lambda B u, \quad \lambda \in R^{1}$.

In $\S 1$, we give a complete self-contained treatment of the existence of minima of functionals on reflexive Banach spaces, a treatment which extends and strengthens earlier studies by Lusternik, E. Rothe, Vainberg, and others (see [6], [11], [12], [14], [15]). In \$2, we apply the results of $\$ 1$ to Problems $A$ and $B$, above. In the case of Problem A, we strengthen and simplify results of Morrey [10] and Smale [13]. The relation of the resulting existence theorem for the solution of the variational boundary-value problem for equation (1) to those obtained by the writer in [2], [3], [4] by operator methods (as well as unpublished results of Leray and Lions) and the results of Višik [16] using other analytical methods, is discussed in detail in [5]. Special cases of the eigenvalue problem treated in Problem $B$ have been treated for $A$ linear by Levinson [7] with $A=\Delta$ on $R^{2}$, and by Berger [1] for general linear $A$.

1. Abstract variational problems. Let $V$ be a real Banach space. Strong convergence in $V$ is denoted by $\rightarrow$, weak convergence by $\rightarrow$. We consider two functions 


$$
\begin{aligned}
& \Phi: V \times V \rightarrow R^{1}, \\
& g: V \rightarrow R^{1},
\end{aligned}
$$

and define $f: V \rightarrow R^{1}$ by $f(v)=\Phi(v, v), v \in V$.

The function $\Phi$ is said to be semi-convex if both of the following conditions hold:

(a) For each $v$ in $V$ and $c$ in $R^{1}$, the subset $W_{c, v}$ of $V$ given by

$$
W_{c, v}=\{u \mid u \in V, \Phi(u, v) \leqq c\}
$$

is convex.

(b) For each bounded set $B$ of $V$ and each sequence $\left\{v_{j}\right\}$ in $V$ with $v_{j} \rightarrow v, \Phi\left(u, v_{j}\right) \rightarrow \Phi(u, v)$ uniformly for $u$ in $B$. For fixed $v$ in $V, \Phi(\cdot, v)$ is continuous in the strong topology of $V$.

THeOREM 1. Let $V$ be a reflexive Banach space, $\Phi$ a semi-convex real function on $V \times V$. Let $f(v)=\Phi(v, v)$ for $v$ in $V$, and let $C$ be a weakly closed bounded subset of $V$. Then $f$ is bounded from below on $C$ and assumes its minimum on $C$.

Proof of Theorem 1 . We may choose a sequence $\left\{u_{j}\right\}$ from the bounded weakly closed set $C$ such that

$$
f\left(u_{j}\right)=\Phi\left(u_{j}, u_{j}\right) \rightarrow c_{0}=\underset{u \in G}{\text { g.l.b. }} f(u),
$$

while

$$
u_{j} \rightarrow u_{0}, \quad u_{0} \in C .
$$

By property (b) of semi-convexity and the boundedness of $C$,

$$
\Phi\left(u_{j}, u_{j}\right)-\Phi\left(u_{0}, u_{j}\right) \rightarrow 0 .
$$

Hence

$$
\Phi\left(u_{0}, u_{j}\right) \rightarrow c_{0} .
$$

Let $c$ be any real number with $c>c_{0}$. Then for $j \geqq j_{c}, u_{j} \in W_{c, u_{0}}$ as defined by equation (3) above. $W_{c, u_{0}}$ is convex by property (a), and is closed by the second part of property (b) of semi-convexity. Hence $W_{c, u_{0}}$ is weakly closed. Since $u_{j} \rightarrow u_{0}, u_{0} \in W_{c, u_{0}}$ i.e., $\Phi\left(u_{0}, u_{0}\right) \leqq c$. Since $c$ was any number $>c_{0}$, it follows that $c_{0}>-\infty$ and $f\left(u_{0}\right)=c_{0}$. Q.E.D.

As corollaries of Theorem 1, we have the following:

THEOREM 2. Let $\Phi$ be a semi-convex real function on $V \times V$, where $V$ is a reflexive $B$-space, and for $v$ in $V$, let $f(v)=\Phi(v, v)$. If $f(v) \rightarrow+\infty$ as $\|v\| \rightarrow+\infty$, then $f$ assumes a minimum on $V$. 
Proof of Theorem 2. Set $C=\{v\|v\| \leqq R\}$ for $R$ sufficiently large.

THEOREM 3. Let $V$ be a reflexive $B$-space, $\Phi$ a real semi-convex function on $V \times V$, and $f(v)=\Phi(v, v)$ for $v$ in $V$. Let $g$ be a weakly continuous real function on $V$. Let $C=\{u \mid g(u)=c\}$ for a fixed $c$ in $R^{1}$ and suppose that $f(u) \rightarrow+\infty$ as $\|u\| \rightarrow+\infty$ on $C$. Then $f$ assumes a minimum on $C$.

Proof of Theorem 3. $C \cap\{u \mid\|u\| \leqq R\}$ is weakly closed and bounded for all $R>0$.

Let $V^{*}$ be the adjoint space of $V,(w, u)$ the pairing between $w$ in $V^{*}$ and $u$ in $V$.

If $g: V \rightarrow R^{1}, g$ is said to be differentiable at $v_{0}$ in $V$ if there exists an element $g^{\prime}\left(v_{0}\right)$ in $V^{*}$ such that for all $h$ in $V$

$$
g\left(v_{0}+h\right)=g\left(v_{0}\right)+\left(g^{\prime}\left(v_{0}\right), h\right)+\epsilon(h)
$$

where $\epsilon(h)=o(\|h\|)$ as $\|h\| \rightarrow 0$. If $\Phi: V \times V \rightarrow R^{1}, \Phi$ is differentiable at $\left(v_{1}, v_{2}\right)$ if there exists a pair $w_{1}, w_{2}$ in $V^{*}$ such that

$\Phi\left(v_{1}+h_{1}, v_{2}+h_{2}\right)=\Phi\left(v_{1}, v_{2}\right)+\left(w_{1}, h_{1}\right)+\left(w_{2}, h_{2}\right)+o\left(\left\|h_{1}\right\|+\left\|h_{2}\right\|\right)$, and we set $w_{1}=\Phi_{1}^{\prime}\left(v_{1}, v_{2}\right), w_{2}=\Phi_{2}^{\prime}\left(v_{1}, v_{2}\right)$. If $\Phi$ is differentiable at $\left(v_{0}, v_{0}\right)$ and $f(v)=\Phi(v, v)$, then $f$ is differentiable at $v_{0}$ and

$$
f^{\prime}\left(v_{0}\right)=\Phi_{1}^{\prime}\left(v_{0}, v_{0}\right)+\Phi_{2}^{\prime}\left(v_{0}, v_{0}\right) \text {. }
$$

THEOREM 4. Let $V$ be a Banach space, $f$ and $g$ two real functions on $V$ with $f$ and $g$ differentiable at $v_{0}, g^{\prime}\left(v_{0}\right) \neq 0$. If $f$ has a local minimum at $v_{0}$ with respect to the set $C=\left\{v \mid g(v)=g\left(v_{0}\right)\right\}$, then there exists $\lambda$ in $R^{1}$ such that $f^{\prime}\left(v_{0}\right)=\lambda g^{\prime}\left(v_{0}\right)$.

Proof of Theorem 4. Let $V_{1}=\left\{v \mid v \in V,\left(g^{\prime}\left(v_{0}\right), v\right)=0\right\}$, and choose $u_{0}$ in $V$ such that $\left(g^{\prime}\left(v_{0}\right), u_{0}\right)=1$. If $v$ is any element of $V_{1}$ with $\|v\|=1$ and $\epsilon$ and $r$ are real numbers with $|\epsilon|,|r|$ sufficiently small, then

$$
f\left(v_{0}\right) \leqq f\left(v_{0}+\epsilon v+r u_{0}\right)
$$

provided that $g\left(v_{0}\right)=g\left(v_{0}+\epsilon_{v}+r u_{0}\right)$. We know that

$$
\begin{aligned}
g\left(v_{0}+\epsilon v+r u_{0}\right) & =g\left(v_{0}\right)+\epsilon\left(g^{\prime}\left(v_{0}\right), v\right)+r\left(g^{\prime}\left(v_{0}\right), u_{0}\right)+s(\epsilon, r, v) \\
& =g\left(v_{0}\right)+r+s(\epsilon, r, v),
\end{aligned}
$$

where for each fixed $v$ in $V_{1}, s(\epsilon, r, v)=o(|\epsilon|+|r|)$. Consider $r$ on the interval $\left[-\frac{1}{2}|\epsilon|,+\frac{1}{2}|\epsilon|\right]$, and the quantity $r+s(\epsilon, r, v)$ with $\epsilon \neq 0$ and $v$ fixed. For $|\epsilon|$ sufficiently small, $r+s(\epsilon, r, v)$ is negative at the left endpoint, positive at the right, and continuous in $r$. We may 
choose a value of $r(\epsilon, v)$ in the interval to make $r+s(\epsilon, r, v)=0$, and hence $|r(\epsilon, v)|=o(|\epsilon|)$. For this choice of $r$, we have

$$
\begin{aligned}
f\left(v_{0}\right) & \leqq f\left(v_{0}+\epsilon v+r u_{0}\right) \\
& =f\left(v_{0}\right)+\epsilon\left(f^{\prime}\left(v_{0}\right), v\right)+r\left(f^{\prime}\left(v_{0}\right), u_{0}\right)+o(|\epsilon|+|r|)
\end{aligned}
$$

so that

$$
\epsilon\left(f^{\prime}\left(v_{0}\right), v\right) \geqq-o(|\epsilon|), \quad|\epsilon| \rightarrow 0 .
$$

Hence $\left(f^{\prime}\left(v_{0}\right), v\right)=0$ for all $v$ in $V_{1}$ and $f^{\prime}\left(v_{0}\right)=\lambda g^{\prime}\left(v_{0}\right)$, for some $\lambda$ in $R^{1}$.

REMARK. In [6] and [14], Theorem 4 is called Lusternik's principle and proofs are given for special cases.

TheOREM 5. Let $V$ be a reflexive Banach space, $\Phi$ a semi-convex real function on $V \times V, g$ a weakly continuous real function on $V$, $f(v)=\Phi(v, v)$ for $v$ in $V$. Suppose that $f$ and $g$ are differentiable on $V$, that for a given constant $c$ in $R^{1}$ the set $C=\{v \mid g(v)=c\}$ is nonempty, and that $g^{\prime}(v) \neq 0$ for $v$ in $C$. Suppose further that $f(v) \rightarrow+\infty$ as $\|v\| \rightarrow+\infty$ on $C$, then there exists $v_{0}$ in $C$ and $\lambda$ in $R^{1}$ such that $f^{\prime}\left(v_{0}\right)$ $=\lambda g^{\prime}\left(v_{0}\right)$.

Theorem 5 is an immediate consequence of Theorems 3 and 4.

A useful complement to Theorem 5 is the following:

THEOREM 6. A sufficient condition for condition (a) for semi-convexity to hold is that $\Phi$ be everywhere differentiable on $V \times V$ and that $\Phi_{1}^{\prime}(u, v)$ be monotone in $u$ for fixed $v$, i.e., for all $u_{0}, u_{1}$ in $V$,

$$
\left(\Phi_{1}^{\prime}\left(u_{1}, v\right)-\Phi_{1}^{\prime}\left(u_{0}, v\right), u_{1}-u_{0}\right) \geqq 0 .
$$

ProOF OF TheOREM 6. Let $u_{0}, u_{1}$, and $v$ be elements of $V, 0 \leqq \lambda \leqq 1$. Let $u_{\lambda}=\lambda u_{1}+(1-\lambda) u_{0}$. To prove condition (a), it suffices to show $\Phi(u, v)$ convex in $u$ for fixed $v$. Set

$$
h(\lambda)=\Phi\left(u_{\lambda}, v\right)-\lambda \Phi\left(u_{1}, v\right)-(1-\lambda) \Phi\left(u_{0}, v\right) .
$$

It suffices to show that $h(\lambda) \leqq 0$ for $0 \leqq \lambda \leqq 1$. Since $h(0)=h(1)=0$, it suffices to show that $h^{\prime}(\lambda)$ is nondecreasing on the interval. However,

$$
h^{\prime}(\lambda)=\left(\Phi_{1}^{\prime}\left(u_{\lambda}, v\right), u_{1}-u_{0}\right)-\Phi\left(u_{1}, v\right)+\Phi\left(u_{0}, v\right),
$$

so that for $\lambda<\xi$,

$$
\begin{aligned}
h^{\prime}(\xi)-h^{\prime}(\lambda) & =\left(\Phi_{1}^{\prime}\left(u_{\xi}, v\right)-\Phi_{1}^{\prime}\left(u_{\lambda}, v\right), u_{1}-u_{0}\right) \\
& =(\xi-\lambda)^{-1}\left(\Phi_{1}^{\prime}\left(u_{\xi}, v\right)-\Phi_{1}^{\prime}\left(u_{\lambda}, v\right), u_{\xi}-u_{\lambda}\right) \geqq 0 . \quad \text { Q.E.D. }
\end{aligned}
$$

REMARK. Connections between monotonicity of the gradient and convexity of the functional have been remarked in Minty [9] and 
implicitly in VaInberg and Kachurovski [15]. Monotone operators between $V$ and $V^{*}$ have been studied in Browder [2], [4] and Minty [8].

2. Nonlinear eigenvalue problems. We adopt the notation of [2] and [3] in general, except that all our functions will be real- rather than complex-valued. Let $\Omega$ be a bounded, smoothly bounded open set in $R^{n}, n \geqq 1, D^{\alpha}$ the elementary differential operator $\left(\partial / \partial x_{1}\right)^{\alpha_{1}} \ldots$ $\left(\partial / \partial x_{n}\right)^{\alpha_{n}}$. We assume that we are given positive integers $r$ and $m$, a real number $p$ with $1<p<\infty$, and a closed subspace $V$ of the reflexive Banach space $W^{m, p}(\Omega)$ of $r$-vector functions $u$ on $\Omega$ such that $D^{\alpha} u \in L^{p}(\Omega)$ for all $\alpha$ with $|\alpha| \leqq m$. Let $\langle$,$\rangle denote the natural inner$ product in $R^{r}$ and for two real-valued $r$-vector functions $u$ and $v$ on $\Omega$, set

$$
[u, v]=\int_{\Omega}\langle u(x), v(x)\rangle d x,
$$

where the integration is taken with respect to Lebesgue $n$-measure.

Let $\zeta=\left\{\zeta_{\alpha}|| \alpha \mid=m\right\}$ and $\psi=\left\{\psi_{\xi}|| \xi \mid \leqq m-1\right\}$ be elements of the real vector spaces $R^{N}$ and $R^{M}$, respectively, where for each $\alpha$ and $\xi, \zeta_{\alpha}$ and $\psi_{\xi}$ are real $r$-vectors. We assume that we are given two functions

$$
F(x, \psi, \zeta), \quad G(x, \psi)
$$

defined on $\Omega \times R^{M} \times R^{N}$ and $\Omega \times R^{M}$, respectively, measurable in $x$ and $C^{1}$ in $(\psi, \zeta)$ or $\psi$. We let $F_{\alpha}, F_{\xi}$, and $G_{\xi}$ denote the appropriate partial gradients of the functions $F$ and $G$ with respect to $\zeta_{\alpha}$ and $\psi_{\xi}$.

We suppose that $F$ and $G$ satisfy the following system of inequalities:

$$
\begin{aligned}
&|F(x, \psi, \zeta)| \leqq c(\eta)\left\{g(x)+|\zeta|^{p}+\sum_{|\xi| \leqq m-1}\left|\psi_{\xi}\right|^{p_{\xi}}\right\}, \\
&|G(x, \psi)| \leqq c(\eta)\left\{g(x)+\sum_{|\xi| \leq m-1}\left|\psi_{\xi}\right| p_{\xi}\right\},
\end{aligned}
$$

where $g \in L^{p}(\Omega), p_{\xi}$ are exponents satisfying the inequalities

$$
(n-p(m-|\xi|)) p_{\xi}<n p \quad \text { if } n-p(m-|\xi|)>0
$$

and $c(\eta)$ is a continuous function of $\eta=\left\{\psi_{\xi}|| \xi \mid<n / p-m\right\}$.

For each $u$ in $V$, let

$$
\zeta(u)=\left\{D^{\alpha} u|| \alpha \mid=m\right\}, \quad \psi(u)=\left\{D^{\xi} u|| \xi \mid \leqq m-1\right\} .
$$

Then the functionals 


$$
\Phi(u, v)=\int_{\Omega} F(x, \psi(v), \zeta(u)) d x
$$

and

$$
g(u)=\int_{\Omega} G(x, \psi(u)) d x
$$

are well defined and continuous on $V \times V$ and $V$, respectively, $g$ is weakly continuous on $V$, and $\Phi$ satisfies condition (b) for semiconvexity. If we assume further that:

$$
\begin{aligned}
\left|F_{\alpha}(x, \psi, \zeta)\right| & \leqq c(\eta)\left\{g_{1}(x)+|\zeta|^{p-1}+\sum_{|\xi| \leq m-1}\left|\psi_{\xi}\right| p^{\prime} \xi\right\}, \\
\left|F_{\beta}(x, \psi, \zeta)\right| & \leqq c(\eta)\left\{g_{1}(x)+|\zeta| e_{\beta}+\sum_{|\xi| \leq m-1}\left|\psi_{\xi}\right| q_{\xi \beta}\right\}, \\
|G(x, \psi)| & \leqq c(\eta)\left\{g_{1}(x)+\sum_{|\xi| \leq m-1}\left|\psi_{\xi}\right| q_{\xi \beta}\right\},
\end{aligned}
$$

where $g_{1} \in L^{p^{\prime-1}}(\Omega)$ and $p_{\xi}^{\prime}, q_{\beta}$, and $q_{\xi \beta}$ are exponents satisfying the inequalities

$$
\begin{aligned}
(n-p(m-|\xi|)) p_{\xi}^{\prime} & \leqq n(p-1), \quad \text { if } n-p(m-|\xi|)>0, \\
(n-p(m-|\xi|)) q_{\xi \beta} & \leqq n(p-1)+p(m-|\beta|), \\
n q_{\beta} & \leqq n(p-1)+p(m-|\beta|)
\end{aligned}
$$

then the functionals $\Phi$ and $g$ are everywhere once differentiable with

$$
\begin{aligned}
\left(\Phi_{1}^{\prime}\left(v_{1}, v_{2}\right), u\right) & =\sum_{|\alpha|=m}\left[F_{\alpha}\left(x, \psi\left(v_{2}\right), \zeta\left(v_{1}\right)\right), D^{\alpha} u\right], \\
\left(\Phi_{2}^{\prime}\left(v_{1}, v_{2}\right), u\right) & =\sum_{|\xi| \leq m-1}\left[F_{\xi}\left(x, \psi\left(v_{2}\right), \zeta\left(v_{1}\right)\right), D^{\xi} u\right], \\
\left(g^{\prime}(v), u\right) & =\sum_{|\xi| \leq m-1}\left[G_{\xi}(x, \psi(v)), D^{\xi} u\right] .
\end{aligned}
$$

If we assume that each $F_{\alpha}$ is itself differentiable in $\zeta$ and that the following semi-ellipticity condition holds:

$$
\sum_{|\alpha|,|\beta|=m}\left\langle F_{\alpha \beta}(x, \psi, \zeta) \eta_{\alpha}, \eta_{\beta}\right\rangle \geqq 0,
$$

for all $\psi$ in $R^{M}, x$ in $\Omega$, and $\zeta$ and $\eta$ in $R^{N}$ (where $F_{\alpha \beta}$ is the gradient of $F_{\alpha}$ with respect to $\zeta_{\beta}$, which we assume to exist); then $\Phi$ will satisfy the monotonicity condition of Theorem 6 and thereby condition (a) for semi-convexity.

Applying Theorem 2, we have: 
THEOREM 7. If $F$ satisfies the inequalities imposed on it in (I), (II), and (III) and if $f(v)=\Phi(v, v) \rightarrow+\infty$ as $\|v\| \rightarrow+\infty$, then $f$ has $a$ minimum on $V$ which is a variational solution in the sense of [2] of the Euler-Lagrange equation

$$
A u=\sum_{|\beta| \leq m}(-1)^{|\beta|} D^{\beta} F_{\beta}(x, \psi(u), \zeta(u))=0 .
$$

Applying Theorem 5, we have:

Theorem 8. If $F$ and $G$ satisfy (I), (II), and (III), if the set $C=\{v \mid v \in V, g(v)=c\}$ is nonempty and $g^{\prime}(v) \neq 0$ on $C$, and if, finally, $f(v) \rightarrow+\infty$ as $\|v\| \rightarrow+\infty$ on $C$, then $f$ has a minimum on $C$ which is a variational solution of the appropriate boundary-value problem for the eigenvalue problem

$$
A u=\lambda B u=\lambda\left\{\sum_{|\xi| \leq m-1}(-1)^{|\xi|} D^{\xi} G_{\xi}(x, \psi(u))\right\} .
$$

We complete our considerations with the following:

Theorem 9. (a) If $V$ is $W_{0}^{m, p}(\Omega)$, the closure of $C_{c}^{\infty}(\Omega)$ in $W^{m, p}(\Omega)$, the boundary conditions in Theorems 7 and 8 are those of the homogeneous Dirichlet problem.

(b) Condition (III) can be replaced by the weaker integral condition

$$
\sum_{|\alpha|,|\beta|-m}\left[F_{\alpha \beta}\left(x, \psi\left(v_{1}\right), \zeta\left(v_{2}\right)\right) D^{\alpha} u, D^{\beta} u\right] \geqq-c\|u\|_{m-1, p}^{p}
$$

(c) Theorem 8 can be specialized to hold under the following more intuitive restrictions than (I) and (II): namely, $|F|<c\left\{1+|\zeta|^{p}+|\psi|^{p}\right\}$, $\left|F_{\alpha}\right|+\left|F_{\xi}\right| \leqq c\left\{1+|\zeta|^{p-1}+|\psi|^{p-1}\right\}, G=u^{q}$ with $q<n p(n-p m)^{-1}$ for $n>p m$ and $G$ an arbitrary continuous function of $u$ for $n<p m$.

The regularity of the solutions of Theorems 7 and 8 can be derived from known results for linear and mildly nonlinear equations $A$ as well as for the case $m=1, r=1$.

\section{BIBLIOGRAPHY}

1. M. Berger, A nonlinear elliptic eigenvalue problem, Dissertation, Yale University, New Haven, Conn., May 1964.

2. F. E. Browder, Nonlinear elliptic boundary value problems, Bull. Amer. Math. Soc. 69 (1963), 862-874. 302.

3. - Nonlinear elliptic problems. II, Bull. Amer. Math. Soc. 70 (1964), 299-

4. - Nonlinear elliptic boundary value problems. II, Trans. Amer. Math. Soc. (to appear). 
5. - Existence and uniqueness theorems for solutions of nonlinear boundary value problems, Proc. Sympos. Appl. Math. Vol. 17, Amer. Math. Soc., Providence, R. I. (to appear).

6. M. A. Krasnoselski, Topological methods in the theory of nonlinear integral equations, GITTL, Moscow,1956 (English transl., Pergamon Press, New York, 1964).

7. N. Levinson, Positive eigenfunctions for $u+\lambda f(u)=0$, Arch. Rational Mech. Anal. 11 (1962), 258-272.

8 G. J. Minty, On a "monotonicity" method for the solution of nonlinear equations in Banach spaces, Proc. Nat. Acad. Sci. U.S.A. 50 (1963), 1038-1041.

9. - On the monotonicity of the gradient of a convex function, Pacific J. Math. 14 (1964), 243-247.

10. C. B. Morrey, Jr., Multiple integral problems in the calculus of variations and related topics, Ann. Scuola Norm Sup. Pisa 14 (1960), 1-61.

11. E. H. Rothe, Gradient mappings and extrema in Banach spaces, Duke Math. J. 15 (1948), 421-431.

12. - A note on the Banach spaces of Calkin and Morrey, Pacific J. Math. 3 (1953), 493-499.

13. S. Smale, Morse theory and a nonlinear generalization of the Dirichlet problem, Ann. of Math. (to appear).

14. M. M. Valnberg, Variational methods for investigation of nonlinear operators, GITTL, Moscow, 1956 (English transl., Holden Day Co., 1964).

15. M. M. VaInberg and R. I. Kachurovski, On the variational theory of nonlinear operators and equations, Dokl. Akad. Nauk SSSR 129 (1959), 1199-1202.

16. M. I. Višik, Quasilinear strongly elliptic systems of differential equations having divergence form, Trudy Moskov. Mat. Obšc. 12 (1963), 125-184.

University of Chicago 